www.nature.com/clinicalpractice/neph

Of the 123 kidney, 44 liver and 29 heart transplant recipients included in the analysis (aged 0.4-18.1 years at transplantation), only two had preoperative scoliosis. At a mean of 9.2 years following transplantation, radiographic evaluation showed that $23 \%$ of the children had a rib or lumbar hump of $6^{\circ}$ or more. Compared with a control group of children from the general population, pediatric transplant recipients had a significantly greater risk of developing scoliosis requiring a brace or operative treatment (RELATIVE RISK 17.0, 95\% cl 6.8-42.7; $P<0.0001)$. Risk of scoliosis was higher in patients who underwent heart transplantation than in patients who underwent kidney or liver transplantation (ODDS RATIO 7.3; $P=0.0001$ ). Growth hormone therapy also increased scoliosis risk (odds ratio 4.0; $P=0.0008$ ).

It is well established that scoliosis occurs frequently in children with congenital heart disease. The high occurrence of scoliosis in pediatric kidney and liver transplant recipients might be a result of immobilization, immunosuppression or metabolic bone disease. In addition, growth hormone therapy-often given to children post-transplantation-rapidly accelerates growth, potentially leading to low bone mineral density and scoliosis.

Rachael Williams

Original article Helenius I et al. (2006) Scoliosis after solid organ transplantation in children and adolescents. Am J Transplant 6: 324-330

\section{GFRs based on cystatin C measurements are accurate in children on renal medication}

Researchers from the Children's Hospital of Eastern Ontario, Canada, have found that various doses of several commonly used renal drugs do not affect cystatin-C-based estimates of pediatric glomerular filtration rate (GFR). This evidence refutes previous findings that certain drugs such as corticosteroids might alter cystatin $\mathrm{C}$ concentrations, thus showing that cystatin $\mathrm{C}$ might well be an accurate indicator of GFR in children with renal disease.

Foster and colleagues retrospectively analyzed 71 children (2.6 months to 18 years of age) with renal disease, who were taking at least one commonly used renal medication such as tacrolimus, ciclosporin, an angiotensinconverting-enzyme inhibitor, losartan, or a corticosteroid. Ratios of directly measured GFR : cystatin-C-based GFR were calculated for each drug at various doses, with a ratio of 1 indicating agreement.

None of the drugs influenced the ratio of measured GFR : cystatin-C-based GFR in a dose-dependent manner. Only the antibacterial drug co-trimoxazole was associated with a GFR ratio significantly $<1 \quad(P=0.045)$, which could have been related to the small number of patients on each study drug. The study did not confirm previous data that showed a dose-dependent increase in cystatin C in adults taking prednisone; however, the authors acknowledge that their study might not have been sufficiently powered to detect a change in cystatin-C-based GFR at low GFRs. Despite these limitations, they support the use of cystatin $\mathrm{C}$ concentration for the calculation of GFR in children.

Pippa Murdie

Original article Foster J et al. (2006) Influence of commonly used drugs on the accuracy of cystatin C-derived glomerular filtration rate. Pediatr Nephrol 21: 235-238

\section{MPGN severity-not subtype- determines outcome}

Membranoproliferative glomerulonephritis (MPGN) is a rare kidney disorder characterized by mesangial cell proliferation and structural changes in glomerular capillary walls. Three subtypes-I, II and III-have been defined by pathologic features; type II disease is thought to be associated with the worst prognosis. Little et al. performed a retrospective analysis to determine which factors predict end-stage renal failure (ESRF) and post-transplantation disease recurrence in MPGN.

The analysis included 70 cases of MPGN diagnosed by renal biopsy in Ireland from 19721995 -30 patients with type I, 23 with type II and 17 with type III. Multivariate analyses showed that higher degree of interstitial fibrosis, higher degree of mesangial proliferation, and presence of $\geq 1$ cellular crescent at diagnosis were independently associated with ESRF $(P=0.04$, $P=0.03$ and $P=0.002$, respectively). Younger age ( $<21$ years) at diagnosis and presence of $\geq 1$ crescent on the initial biopsy were associated with disease recurrence in the renal allograft $(P=0.04$ and $P=0.005$, respectively). MPGN subtype was not associated with risk of recurrence

\section{GLOSSARY}

RELATIVE RISK

The probability that those with, relative to those without, a risk factor or exposure will experience an outcome (e.g. disease or death)

\section{CONFIDENCE INTERVAL} (Cl)

An estimated range of values (based on a given set of sample data) that has a specified probability of containing the value being estimated

\section{ODDS RATIO}

Ratio of odds of an event in intervention group to odds in control group; when $<1$ for an undesirable outcome, the intervention reduced the risk 